\title{
Efecto del agua tratada magnéticamente en el desarrollo y la producción de cúrcuma (Curcuma longa L.)
}

\author{
Effect of magnetically treated water on the growth \\ and production of curcuma (Curcuma longa L.)
}

ORLANDO ZÚÑIGA'

JHONY ARMANDO BENAVIDES 1,3

CRISTIAN ORLANDO JIMÉNEZ1

MAURICIO ALEJANDRO GUTIÉRREZ1

CELINA TORRES ${ }^{2}$

Plantas de cúrcuma de tres

meses de edad.

Foto: J.A. Benavides

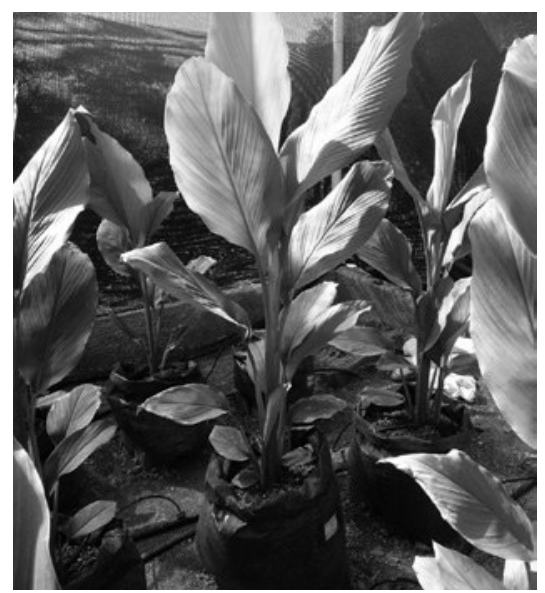

\section{RESUMEN}

La cúrcuma es un cultivo promisorio para la región del Valle del Cauca (Colombia), el cual demanda una alta disponibilidad del recurso hídrico para obtener rendimientos óptimos. La irrigación es un factor fundamental para lograr tales rendimientos, por tal motivo una de las tareas más importantes es la de asegurar la disponibilidad de agua en todo su ciclo vegetativo. Con el propósito de evaluar nuevas tecnologías que contribuyan al mejoramiento de la producción de este cultivo, este trabajo examinó la respuesta del agua tratada magnéticamente (ATM) en el desarrollo fisiológico y la producción de la cúrcuma. Para la estimulación del agua se utilizaron dos magnetizadores instalados en serie dentro de un circuito de recirculación. En las pruebas se establecieron tres tiempos de exposición al campo magnético $(10,15$ y $30 \mathrm{~min})$, un testigo recirculado sin magnetizadores y un testigo absoluto sin recircular. Se empleó un diseño unifactorial completamente al azar. El sistema de magnetización fue acoplado a un sistema de riego por goteo, aplicándose durante tres meses a plántulas de cúrcuma en condiciones de invernadero. Los resultados mostraron que el tiempo de recirculación del ATM durante 30 min con una inducción magnética de $156 \mathrm{mT}$ generó un incremento estadísticamente significativo en la longitud de la planta, el número de rizomas, el número de macollos, la masa fresca y la masa seca, superior a los demás tratamientos. De acuerdo a estos resultados, la aplicación de ATM podría ser considerada como una tecnología promisoria para mejorar el rendimiento del cultivo de cúrcuma.

1 Facultad de Ciencias Naturales y Exactas, Departamento de Física, Grupo de Investigación en Ciencias Ambientales y de la Tierra (ILAMA), Universidad del Valle, Santiago de Cali (Colombia).

2 Facultad de Ciencias Naturales y Exactas, Departamento de Biología, Grupo de Investigación en Biología de Plantas y Microorganismos, Universidad del Valle, Santiago de Cali (Colombia).

3 Autor para correspondencia: jhony.benavides@correounivalle.edu.co 


\section{ABSTRACT}

Curcuma longa is a promising crop for the region of Valle del Cauca (Colombia), which demands a high availability of water resources in order to obtain optimum yields. Irrigation is a key factor in achieving such yields; for that reason, one of the more important tasks is to ensure the availability of water throughout the vegetative cycle. With the aim of evaluating new technologies that contribute to the improvement of the production of this crop, this paper examined the effect of magnetically treated water (MTW) on the physiological growth and production of $C$. longa. For the water stimulation, two magnetizers, installed in series within a recirculation circuit, were used. Three exposure times to the magnetic field were used (10, 15 and $30 \mathrm{~min}$ ), along with a recirculated control treatment without magnetrons and an absolute control treatment without recirculation. The experiment design was completely randomized with one factor. The magnetization system was attached to a drip irrigation system; the treatments were applied for three months to $C$. longa plants under greenhouse conditions. The results showed that the recirculation time of MTW for $30 \mathrm{~min}$ with a magnetic induction of $156 \mathrm{mT}$ resulted in a statistically significant increase in the length of the plants, the number of rhizomes, the number of tillers, the fresh weight and the dry weight, higher than that of the other treatments. According to these results, the application of magnetized water could be regarded as a promising agro-environmental technology that improves crop yield in C. longa.

Additional keywords: bio-magnetism, agro-environmental technology, magnetic field.

Fecha de recepción: 21-11-2015

Aprobado para publicación: 14-05-2016

$\longrightarrow$

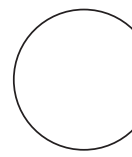

INTRODUCCIÓN

La dependencia de fertilizantes de síntesis química para la producción agrícola competitiva es una de las mayores dificultades que enfrenta la agricultura convencional. Según el documento Conpes 3577 (2009), se acepta que los fertilizantes son un componente importante en los costos de producción de la actividad agropecuaria, afectando directamente el precio de comercialización de los productos y el margen de ganancia del agricultor. El precio que debe pagar un agricultor por fertilizantes minerales en una zona remota es mucho más alto que el precio del mercado mundial a causa del transporte, distribución y otros costos de transacción asociados
(Kotschi, 2015), lo que en muchas ocasiones no se traduce en un mayor valor de compra para el agricultor (Pérez, 2014). En este sentido se hace necesario impulsar el desarrollo de tecnologías innovadoras que propicien la disminución del uso de fertilizantes minerales a la vez que mejoren la eficiencia en el uso del agua de riego y las condiciones del suelo para los cultivos.

La tecnología evaluada en la presente investigación corresponde al uso del campo magnético sobre agua de riego. La tecnología del ATM genera efectos incrementales sobre el crecimiento y desarrollo de las plantas. Esto es debido a que los 
cambios en las propiedades físicas y químicas del agua tratada magnéticamente (ATM) alteran directa o indirectamente el crecimiento y desarrollo de las plantas. Varios autores han encontrado que la exposición previa del agua de riego a un campo magnético (CM) conduce a un aumento de la productividad vegetal y a ciertos cambios en su fisiología (Maheshwari y Grewal, 2009; Al-Khazan et al. 2011; Abou El-Yazied et al. 2012; Hozayn et al. 2013; Mahmood y Usman, 2014).

Un CM aplicado al agua de riego mejora las características de crecimiento de las plantas (Maheshwari y Grewal 2009), la funcionalidad de las raíces (Aladjadjiyan, 2010), además, tiene una marcada influencia en la composición química de las plantas (Radhakrishnan y Kumari, 2012), afecta la disponibilidad de nutrientes en el suelo (Maheshwari y Grewal, 2009) y activa las enzimas de las plantas (Alikamanoglu y Sen, 2011) influyendo, por tanto, en su temprana producción y mejora de rendimiento. Consecuentemente, la aplicación de CM puede ser usada como una alternativa a los métodos químicos de tratamiento de plantas para mejorar la eficiencia en la producción.

Se ha encontrado que el tratamiento magnético del agua tiene un efecto positivo en el mejoramiento de la calidad de la misma. Maheshwari y Grewal (2009) reportaron que dicho tratamiento amortigua los efectos deletéreos de agua reciclada de una planta de tratamiento de aguas residuales (PTAR) y de agua salina, usadas para riego agrícola con 1.500 ppm y 3.000 ppm de $\mathrm{NaCl}$ aplicadas a plantas de apio (Apium graveolens) y fríjol (Phaseolus vulgaris), respectivamente y con un campo magnético de 3,5 a $136 \mathrm{mT}$. El rendimiento y productividad en plantas de apio $y$ de fríjol cultivadas con este tipo de aguas se vio incrementado casi al nivel de aquellas regadas con agua de calidad, y por encima de las que solo recibieron agua sin tratamiento magnético, bajo condiciones controladas de invernadero. Estos resultados son congruentes con lo reportado por Marei et al. (2014) quienes al emplear agua salina para riego tratada con CM evidenciaron un incremento importante en el rendimiento y en la eficiencia en el uso del agua (EUA) en un cultivo de ají (Capsicum annuum L.).

Khoshravesh et al. (2011) investigaron los efectos del ATM y la salinidad del agua de riego en la distribución de la humedad sobre el suelo bajo riego por goteo. Ellos demostraron que la humedad media del suelo, a diferentes profundidades del mismo, bajo el sistema de goteo con el tratamiento de ATM, fue mayor en comparación con el tratamiento de riego con agua sin magnetizar con una diferencia significativa del 5\%. Hilal et al. (2013) indican que el tratamiento magnético de agua para riego agrícola dependerá de la intensidad del campo magnético, la composición de las sales disueltas y la velocidad en que cruza la fuente del campo.

Hozayn et al. (2013) indicaron que la aplicación de ATM en un cultivo de remolacha azucarera indujo incrementos significativos en el rendimiento de raíces (masa, longitud y diámetro) en 21,$53 ; 5,68$ y $16,23 \%$ respectivamente, al ser comparado con el agua sin magnetizar. Los resultados obtenidos por diferentes autores confirman los efectos benéficos de campos magnéticos de baja frecuencia sobre el crecimiento de las raíces y las hojas de la remolacha azucarera (Vasilevski, 2003; Rochalska et al., 2008).

Hozayn et al. (2011) luego de regar cultivos de trigo, lino, garbanzo y lenteja con ATM, encontraron que todas las plantas exhibieron un incremento notable en el desarrollo vegetativo y en sus constituyentes bioquímicos. Además, los resultados obtenidos indican que el número de bandas proteínicas aumentó con el uso de ATM en comparación con la no tratada. Además de lo anterior, el tratamiento con ATM incrementó los rendimientos de todos los cultivos evaluados. Los incrementos de rendimiento de semilla/planta en los cultivos de monocotiledóneas alcanzaron el 10 y 33,33\% para el lino y el trigo respectivamente, y en los cultivos de dicotiledóneas se al- 
canzaron valores de 26,92 y 46,62\%, para lenteja y garbanzo, respectivamente comparados con los cultivos regados con agua no tratada.

La heterogeneidad de las especies y tipos de plantas, condiciones físico-químicas del agua utilizada para riego agrícola, tipos de suelo, condiciones locales y la falta de un modelo físico así como de unos protocolos estandarizados para el tratamiento de la tecnología de magnetización con fines agrícolas es muy compleja, de ahí que sean necesarias más investigaciones a nivel local para obtener información suficiente como para generar curvas detalladas que integren la intensidad del campo, el tiempo de exposición y la respuesta en cultivos promisorios como lo es el de la cúrcuma sobre unidades y frecuencias de riego definidas y diferenciadas.

El objetivo de este trabajo de investigación estuvo encaminado a evaluar la respuesta del cultivo de la cúrcuma bajo condiciones de invernadero a la aplicación de la tecnología de ATM y determinar el tiempo de exposición óptimo de recirculación para obtener mejores respuestas agronómicas en un cultivo de cúrcuma.

\section{MATERIALES Y MÉTODOS}

La investigación se realizó bajo condiciones de invernadero en el vivero ILAMA ubicado en la granja experimental de la Escuela de Ingeniería de Recursos Naturales y del Ambiente EIDENAR de la Universidad del Valle, Cali, la cual se encuentra a una altitud de 995 msnm y ubicada a $3^{\circ} 22^{\prime} 33,55^{\prime \prime} \mathrm{N}$ y $76^{\circ} 31^{\prime} 58,43$ ”W; cuenta con una temperatura promedio anual de $23^{\circ} \mathrm{C}$, humedad relativa del $73 \%$, precipitación promedio anual de 908 mm y 162 h en promedio de brillo solar.

\section{Aplicación del tratamiento magnético}

El sistema de recirculación para agua de riego cuenta con dos magnetizadores marca Quantum Biotek modelo Omni Water System de 2,54 cm de diámetro conectados en serie. La intensidad del campo se midió con el teslámetro LD Didactic $\mathrm{GmbH}$ (Huerth, Alemania) reportando una inducción magnética de 156 militeslas (mT) y una electrobomba Titán modelo QB60 (Taizhou Ouke Pump, Zhejiang, China) con caudal máximo de 36 litros por minuto usada para impulsar el agua en el circuito. La inducción magnética se estableció luego de revisar varias investigaciones que utilizan valores intermedios (entre 90 y 250 $\mathrm{mT}$ ) con resultados interesantes (De Souza et al., 2005, 2006; Flórez et al., 2007; Racuciu et al., 2008; Domínguez-Pacheco et al., 2010; Vashisth et al., 2010; Bhardwaj et al., 2012). Para el almacenamiento del agua se utilizó un recipiente plástico de 100 L y un sistema de tubería de PVC de 2,54 cm de diámetro con cinco válvulas de control de flujo (figura 1).

\section{Diseño experimental}

El ensayo se ajustó a un diseño unifactorial de múltiples niveles dispuestos completamente al azar con el factor: tiempo de recirculación a un campo magnético constante (156 mT) y tres ni-

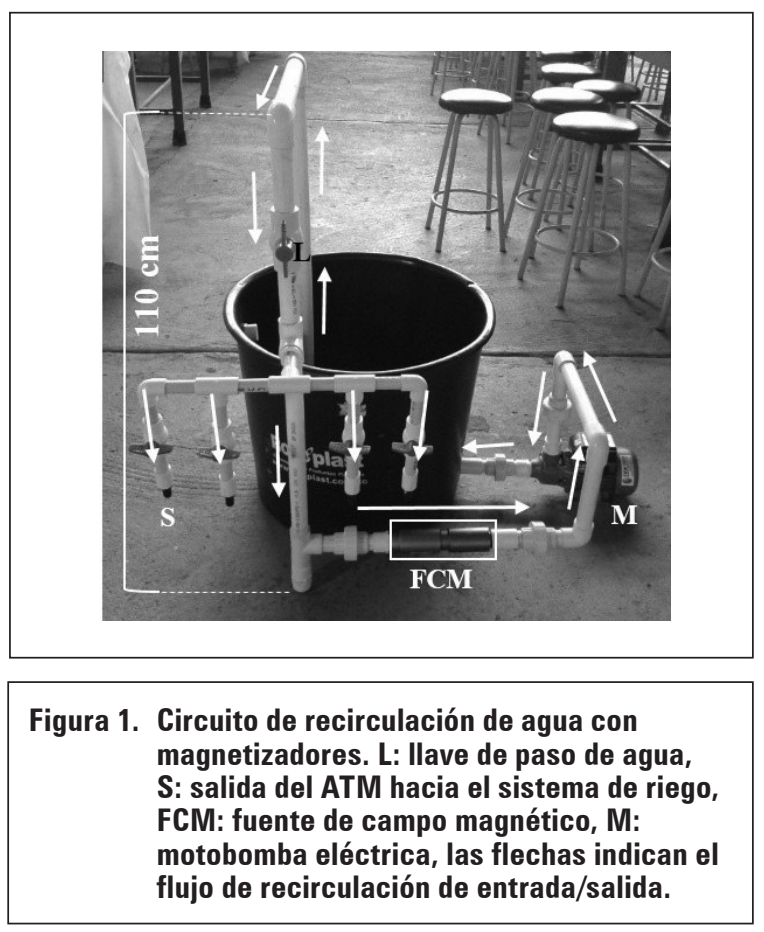


veles (10, 15 y 30 min) de recirculación. Se contó con un tratamiento testigo $(\mathrm{T})$ que fue recirculado sin los magnetizadores acoplados al sistema y un testigo absoluto ( $\mathrm{Ta}$ ) que no fue recirculado ni magnetizado y se obtuvo de un sistema de riego alterno.

Para el desarrollo del montaje experimental se preseleccionaron rizomas de Curcuma longa L., provenientes de la vereda El Limonar en el municipio de Dagua, Valle del Cauca, Colombia. Las unidades experimentales para todos los tratamientos consistieron en plántulas de cúrcuma que se pregerminaron en arena de río durante un mes. Se dispusieron tres réplicas para el Ty el Ta y cinco réplicas para los tres tratamientos restantes, obteniendo un total de 21 unidades experimentales.

Una vez las plántulas desarrollaron una hoja verdadera, se trasplantaron en bolsas plásticas, con $15 \mathrm{~kg}$ de suelo proveniente de la vereda El Limonar, municipio de Dagua Valle del Cauca. En la tabla 1 se exponen los resultados de la caracterización físico-química del suelo utilizado

Para la aplicación de los tratamientos se implementó un sistema de riego localizado de alta frecuencia, que permitió la aplicación inmediata del agua después de la magnetización. Se utilizó manguera de $16 \mathrm{~mm}$ para conducir el agua desde el dispositivo de estimulación hasta las plantas de cúrcuma, donde se aplicó utilizando goteros con caudal medio de $2 \mathrm{~L} \mathrm{~h}^{-1}$. Se regó con una frecuencia fija de aplicación de 3 días.

La cantidad de agua aplicada resultó del cálculo de la demanda hídrica, teniendo en cuenta las condiciones climáticas de una estación cercana del Centro Internacional de Agricultura Tropical (CIAT). Utilizando los software Climwat y Cropwat de la FAO, fue posible determinar la evapotranspiración de referencia $\left(E T_{\mathrm{r}}\right)$ crítica, con un valor de $3,8 \mathrm{~mm} \mathrm{día}^{-1}$. Y posteriormente se determinó la evapotranspiración real del cultivo $\left(\mathrm{ET}_{\mathrm{c}}\right)$ de cúrcuma a partir de la ecuación 1.

$$
\mathrm{ETc}=\mathrm{ETr} * \mathrm{Kc}
$$

Donde $\mathrm{K}_{\mathrm{c}}$ es el coeficiente del cultivo, que posee diferentes valores según la etapa fisiológica del cultivo. La demanda hídrica expresada en necesidades diarias $\left(\mathrm{N}_{\mathrm{d}}\right)$ en $\mathrm{L}$ día ${ }^{-1}$, resultó del cálculo según la ecuación 2.

$\mathrm{Nd}=\frac{\mathrm{ET}_{\mathrm{c}} * \mathrm{~A}_{\mathrm{h}}}{\mathrm{E}_{\mathrm{a}} * \mathrm{CU}}$

Donde, Ah es el área de humedecimiento, $\mathrm{E}_{\mathrm{a}}$ la eficiencia de aplicación y CU el coeficiente de uniformidad.

Se evaluaron las siguientes variables de respuesta: longitud de la planta $(\mathrm{cm})$, número de rizomas, número de macollos, masa fresca (g) y masa seca (g). Después de $7 \mathrm{~d}$ de trasplante se tomaron mediciones de longitud de la planta. Las mediciones de longitud de la planta se realizaron cada 2 a 5 d, tomando en consideración la distancia entre el nivel del suelo de la matera hasta el punto de inserción de la hoja central más joven. La cosecha se llevó a cabo 109 d después de la siembra. La masa fresca se midió con la balanza analítica Ohaus Traveler (Ohaus, Parsippany, NJ), posteriormente, las plantas se trocearon y se empacaron por separado en bolsas de papel. Las bolsas se guardaron durante $7 \mathrm{~d}$ a una temperatura de $90^{\circ} \mathrm{C}$ en el horno 40GC de Quincy Lab. (Chicago, IL) garantizando así un porcentaje de hume-

Tabla 1. Caracterización físico-química del suelo utilizado.

\begin{tabular}{|c|c|c|c|c|c|c|c|c|c|c|c|c|c|c|c|}
\hline Arena & Limo & Arcilla & $\mathrm{CO}$ & \multirow[t]{2}{*}{ Textura } & \multirow[t]{2}{*}{$\mathrm{pH}$} & CE & $P$ & $S$ & $\mathrm{Fe}$ & K & $\mathrm{Ca}$ & $\mathrm{Mg}$ & $\mathrm{Na}$ & $\mathrm{Al}$ & CICE \\
\hline \multicolumn{4}{|c|}{$\%$} & & & $\mathrm{dS} \mathrm{m}^{-1}$ & \multicolumn{3}{|c|}{$\mathrm{mg} \mathrm{kg}^{-1}$} & \multicolumn{6}{|c|}{$\mathrm{cmol}(+) \mathrm{kg}^{-1}$} \\
\hline 40 & 22 & 38 & 2,4 & $\mathrm{Fr}-\mathrm{Ar}$ & 5,2 & 0,12 & 1,3 & 7,2 & 150 & 0,2 & 3,8 & 3,6 & 0,2 & 0,07 & 7,9 \\
\hline
\end{tabular}


dad cercano al 10\%. Se registró la masa seca con la balanza analítica Ohaus Traveler.

\section{Análisis estadístico}

Se realizó un análisis de varianza a los datos obtenidos con un nivel de confianza del $95 \%$ y una vez verificada, se siguió con la comparación entre medias con el test de Tukey $(\alpha=0,05)$ con el paquete estadístico Minitab versión 16.

\section{RESULTADOS Y DISCUSIÓN}

\section{Longitud de la planta}

El tratamiento $156 \mathrm{mT}-30$ min presentó los mejores resultados en todas las mediciones realizadas en el periodo de evaluación. Este tratamiento superó al Ta y al T en 48,9\% y 44,9\%, respectivamente. Con respecto al tratamiento $156 \mathrm{mT}-10$ min y al de 156 mT-15 min, el tratamiento 156 mT-30 min los superó en 37,4\% y en 38,4\%, respectivamente.

El efecto de la aplicación de campo magnético al agua contribuye al aumento de la disponibilidad de nutrientes disueltos. El-Kholy et al. (2015) analizaron el efecto del ATM sobre un cultivo de banano Williams (Musa sp.) con dos niveles diferentes de nitrógeno $(\mathrm{N})$, fósforo $(\mathrm{P})$ y potasio (K) (100 y $80 \%$ de la dosis recomendada) encontrando un incremento positivo en todos los parámetros de estudio con una tendencia similar con la dosis recomendada en comparación con las plantas regadas con agua sin tratar; por su parte Hilal et al. (2013) encontraron que el uso de agua de riego magnetizada incrementó significativamente las concentraciones de $\mathrm{K}^{+}$sobre el suelo en comparación al testigo, donde la mayor concentración de $\mathrm{K}^{+}$se halló a una profundidad de 15 a $30 \mathrm{~cm}$ mientras que las menores concentraciones se encontraron en la superficie del suelo $(0-5 \mathrm{~cm})$.

La toma de nutrientes por la cúrcuma tiene el siguiente orden: $\mathrm{K}>\mathrm{N}>\mathrm{Mg}>\mathrm{Ca}$ (Rethinam et al., 1994) y la producción promedio de rizomas secos de 5,5 t ha-1 remueve $91 \mathrm{~kg}$ de $\mathrm{N}, 16,9 \mathrm{~kg}$ de $\mathrm{P}_{2} \mathrm{O}_{5}$ y $245 \mathrm{~kg}$ de $\mathrm{K}^{+}$(Sadanandan et al., 1996), lo que demuestra que este cultivo consume altas cantidades de $\mathrm{K}^{+}$para su desarrollo fisiológico. El suelo contó con niveles medios de $\mathrm{K}^{+}$(Rojas et al., 1992; Andrades y Martínez, 2014) (tabla 1), no obstante, fue con el tratamiento $156 \mathrm{mT}$ 30 min con el que la planta alcanzó una mayor altura con respecto a los demás tratamientos (figura 2), esto podría indicar un aumento en la disponibilidad del $\mathrm{K}^{+}$debido al riego con ATM, hecho que concuerda con resultados obtenidos por Mahmood y Usman (2014).

\section{Número de rizomas}

El tratamiento que mostró la mayor diferencia estadísticamente significativa $(P \leq 0,05)$ en el número de rizomas corresponde al tratamiento 156 mT-30 min que superó al Ta y al T en un 126 y $171,2 \%$, respectivamente (tabla 2 ).

Promedios con letras distintas, en la misma columna, indican diferencia significativa según la prueba de Tukey $(P \leq 0,05)$.

\section{Número de macollos}

Para el número de macollos, el tratamiento 156 mT-30 min superó $(P \leq 0,05)$ al Ta y al $\mathrm{T}$ en un 90,9 y $110 \%$ respectivamente (tabla 2 ). Hozayn et al. (2011) sugirieron que el tratamiento magnético sobre agua de riego promueve la absorción de N, Py K e incrementa el número de raíces, el grosor de tallo y número de macollos, lo cual guarda concordancia con los resultados obtenidos.

Para que la cúrcuma generara un buen número de macollos, la planta debía estar sana, a este respecto, Moussa (2011) al evaluar el efecto del ATM a una intensidad de $30 \mathrm{mT}$ aplicada sobre un cultivo de fríjol común (Phaseolus vulgaris), concluyó que esta puede mejorar la cantidad y calidad del cultivo, al estimular el sistema de defensa, la actividad fotosintética y la eficiencia de 


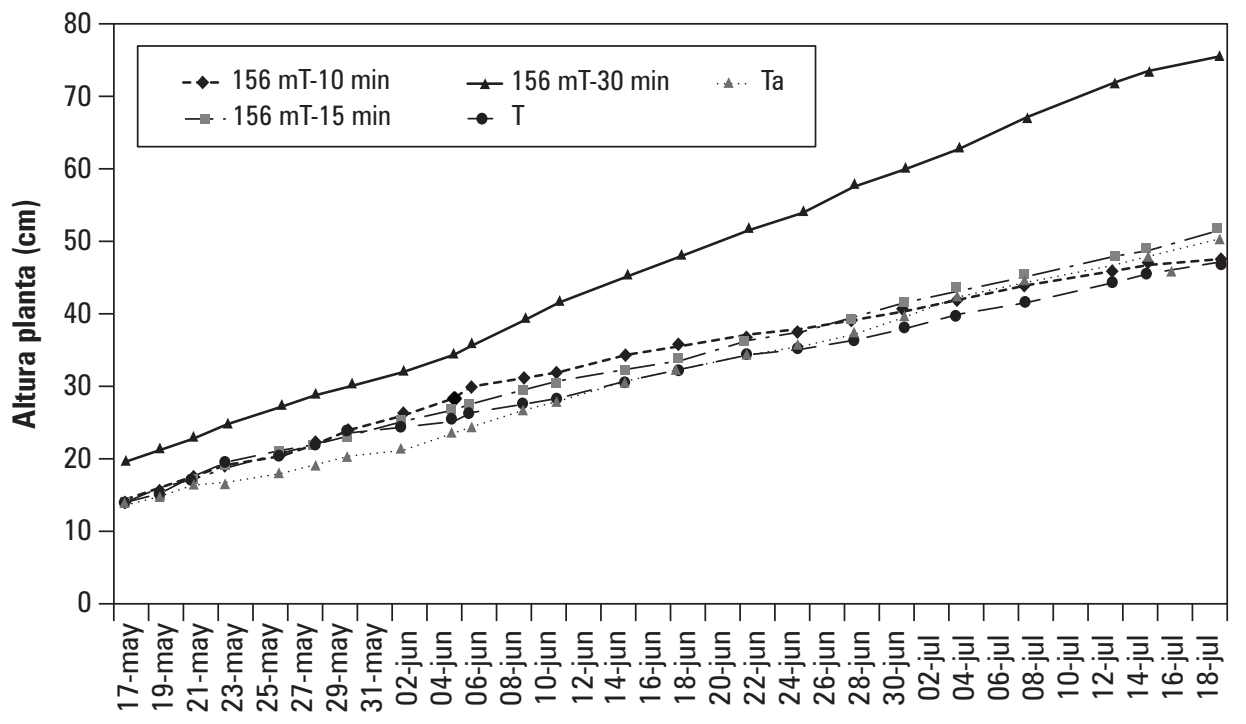

Tiempo (días)

Figura 1. Altura de planta de cúrcuma vs. tiempo.

Tabla 2. Efecto de los tratamientos sobre las variables de respuesta bajo condiciones de invernadero.

\begin{tabular}{|c|c|c|c|c|c|c|}
\multirow{2}{*}{ Tratamientos } & $\begin{array}{c}\text { Niveles del } \\
\text { tratamiento }\end{array}$ & $\mathbf{N}$ & $\begin{array}{c}\text { Número de } \\
\text { rizomas }\end{array}$ & $\begin{array}{c}\text { Número de } \\
\text { macollos }\end{array}$ & $\begin{array}{c}\text { Masa fresca } \\
(\mathrm{g})\end{array}$ & $\begin{array}{c}\text { Masa seca } \\
(\mathrm{g})\end{array}$ \\
\hline \multirow{3}{*}{ Tiempo de exposición (min) } & 30 & 5 & $22,6 \mathrm{a}$ & $7 \mathrm{a}$ & $984,18 \mathrm{a}$ & $85,64 \mathrm{a}$ \\
\cline { 2 - 7 } & 15 & 5 & $14,8 \mathrm{~b}$ & $5 \mathrm{~b}$ & $761,84 \mathrm{~b}$ & $72,32 \mathrm{ab}$ \\
\cline { 2 - 7 } & 10 & 5 & $13,8 \mathrm{bc}$ & $4,8 \mathrm{~b}$ & $739,5 \mathrm{~b}$ & $72,3 \mathrm{ab}$ \\
\hline $\mathrm{Ta}$ & & 3 & $10 \mathrm{bc}$ & $3,6 \mathrm{bc}$ & $548,3 \mathrm{c}$ & $55,37 \mathrm{~b}$ \\
\hline $\mathrm{T}$ & & 3 & $8,3 \mathrm{c}$ & $3,3 \mathrm{c}$ & $489,37 \mathrm{c}$ & $51,97 \mathrm{~b}$ \\
\hline
\end{tabular}

Promedios con letras distintas, en la misma columna, indican diferencia significativa según la prueba de Tukey $(P \leq 0,05)$.

translocación de los fotoasimilados en la planta, dando como resultado el desarrollo de plantas de mayor tamaño y con producciones de rizomas superiores al testigo absoluto.

\section{Masa fresca y seca}

Tanto la masa fresca como la masa seca presentaron los mejores resultados $(P \leq 0,05)$ con el tratamiento $156 \mathrm{mT}-30 \mathrm{~min}$. Para la masa fresca, el tratamiento con mayor diferencia significativa superó n al Ta y al T en 101,1 y 79,5\%, respectivamente, mientras que para la masa seca, el tratamiento $156 \mathrm{mT}-30 \mathrm{~min}$ superó en promedio al Ta y al T en 54,7 y $64,8 \%$, respectivamente.

Una de las posibles explicaciones del efecto positivo observado en el tratamiento magnético se halla en las propiedades paramagnéticas de los átomos en las células y pigmentos de las plantas, es decir, los cloroplastos. En un campo magnético externo, los momentos magnéticos de estos 
átomos se alinean con la dirección del campo. Las propiedades magnéticas de las moléculas determinan su habilidad de absorber la energía del campo magnético, para luego transformarla en otro tipo de energía y transferirla luego a otras estructuras en las células de la planta, generando así su activación. Los efectos magnéticos en plantas pueden ser explicados con la estructura de la resonancia del ciclotrón de iones y los modelos de radicales pares, dos mecanismos que también juegan un importante papel en la magnetorrecepción de otros organismos (Galland y Pazur, 2005).

\section{CONCLUSIONES}

El mejor resultado obtenido sobre las variables de respuesta analizadas (altura de la planta, número de rizomas, número de macollos, masa fresca y masa seca) fue obtenido con ATM con una inducción magnética de 156 mT durante 30 min.

La óptima mitigación de los detrimentos que causan factores adversos al emplearse ATM abre una puerta a su uso extendido en la agricultura. En particular, el tratamiento magnético de aguas de riego es una alternativa aplicable en Colom- bia, donde se presentan condiciones de baja calidad del agua, presencia de sales e inmovilidad de nutrientes en el suelo. Adicionalmente, esta tecnología podría optimizar el uso del agua en cultivos, ya que amortigua los efectos de una oferta hídrica limitada.

Si bien hacen falta más investigaciones a nivel local y nacional que den cabal explicación acerca de la interacción ATM-suelo-planta, el conocimiento actual permite presentar a la tecnología de ATM como una alternativa con altas potencialidades para la producción agrícola.

\section{AGRADECIMIENTOS}

La presente investigación fue desarrollada con recursos provenientes del Sistema General de Regalías en el marco del proyecto "Desarrollo de un Sistema Agroindustrial Rural Competitivo en una Bioregión del Valle del Cauca" a cargo del Grupo de Investigación en Ciencias Ambientales y de la Tierra ILAMA, adscrito a la Facultad de Ciencias Naturales y Exactas de la Universidad del Valle, sede Meléndez, Cali, Colombia.

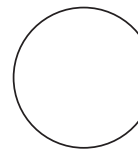

Abou El-Yazied, A., A.M. El-Gizawy, S.M. Khalf, A. El-Satar y O.A. Shalaby. 2012. Effect of magnetic field treatments for seeds and irrigation water as well as N, P and K levels on productivity of tomato plants. J. Appl. Sci. Res. 8(4), 2088-2099.

Aladjadjiyan, A. 2010. Influence of stationary magnetic field on lentil seeds. Int. Agrophys. 24(3), 321-324.

Alikamanoglu, S. y A. Sen. 2011. Stimulation of growth and some biochemical parameters by magnetic field in wheat (Triticum aestivum L.) tissue cultures. Afr. J. Biotechnol. 10(53), 10957-10963. Doi: 10.5897/AJB11.1479
REFERENCIAS BIBLIOGRÁFICAS

Al-Khazan, M., M.B. Abdullatif y N. Al-Assaf. 2011. Effects of magnetically treated water on water status, chlorophyll pigments and some elements content of Jojoba (Simmondsia chinensis L.) at different growth stages. Afr. J. Environ. Sci. Tech. 5(9), 722-731. Doi: 10.5897/AJEST11.117

Andrades, M. y M.E. Martínez. 2014. Fertilidad del suelo y parámetros que la definen. $3^{a}$ ed. Servicio de Publicaciones, Universidad de la Rioja, La Rioja, España.

Bhardwaj, J., A. Anand y S. Nagarajan. 2012. Biochemical and biophysical changes associated with magnetopriming in germinating cucumber seeds. 
Plant Physiol. Biochem. 57, 67-73. Doi: 10.1016/j. plaphy.2012.05.008

CONPES. 2009. Documento CONPES 3577. Política nacional para la racionalización del componente de costos de producción asociado a los fertilizantes en el sector agropecuario. En: Asociación Nacional de Empresarios de Colombia (ANDI), http://www.andi.com.co/es/PC/SobProANDI/ Documentos\%20Sobre\%20Procultivos\%20ANDI/ Conpes\%20-\%20Fertilizantes-3577-18-\%20Marzo-2009.pdf; consulta: Abril de 2016.

De Souza, A., D. García, L. Sueiro, L. Licea y E. Porras. 2005. Pre-sowing magnetic treatment of tomato seeds: effects on the growth and yield of plants cultivated late in the season. Span. J. Agric. Res. 3(1), 113-122. Doi: 10.5424/sjar/2005031-131

De Souza, A., D. García, L. Sueiro, F. Gilart, L. Licea y E. Porras. 2006. Pre-sowing magnetic treatments of tomato seeds increase the growth and yield of plants. Bioelectromagnetics 27(4), 247-257. Doi: 10.1002/bem.20206

Domínguez-Pacheco, A., C. Hernández-Aguilar, A. Cruz-Orea, A. Carballo-Carballo, R. Zepeda-Bautista y E. Martínez-Ortíz. 2010. Semilla de maíz bajo la influencia de irradiación de campos electromagnéticos. Rev. Fitotecn. Mex. 33(2), 183-188.

El-Kholy, M.F., S. Samia y A.A. Farag. 2015. Effect of magnetic water and different levels of NPK on growth, yield and fruit quality of Williams banana plant. Nat. Sci. 13(7), 94-101.

Flórez, M., M.V. Carbonell y E. Martínez. 2007. Exposure of maize seeds to stationary magnetic fields: Effects on germination and early growth. Environ. Exp. Bot. 59(1), 68-75. Doi: 10.1016/j.envexpbot.2005.10.006

Galland, P. y A. Pazur. 2005. Magnetoreception in plants. J. Plant Res. 118(6), 371-389. Doi: 10.1007/ s10265-005-0246-y

Hilal, M.H., Y.M. El-Fakhrani, S.S. Mabrouk, A.I. Mohamed y B.M. Ebead. 2013. Effect of magnetic treated irrigation water on salt removal from a sandy soil and on the availability of certain nutrients. Int. J. Eng. Appl. Sci. 2(2), 36-44.

Hozayn, M., A.A. Abd El Monem, A.M.S. Abdul Qados y E.M. Abd El-Hameid. 2011. Response of some food crops to irrigation with magnetized water under greenhouse condition. Aust. J. Basic Appl. Sci. 5(12), 29-36.

Hozayn, M., A.A. Abd El Monem, R.E. Abdelraouf y M. Abdalla. 2013. Do magnetic water affect wa- ter efficiency, quality and yield of sugar beet (Beta vulgaris L.) plant under arid regions conditions? J. Agron. 12(1), 1-10. Doi: 10.3923/ja.2013.1.10

Khoshravesh, M., B. Mostafazadeh-Fard, S.F. Mousavi y A.R. Kiani. 2011. Effects of magnetized water on the distribution pattern of soil water with respect to time in trickle irrigation. Soil Use Manage. 27(4), 515-522. Doi: 10.1111/j.1475-2743.2011.00358.x

Kotschi, J. 2015. A soiled reputation. Adverse impacts of mineral fertilizers in tropical agriculture. Heinrich Böll Foundation Publisher, Berlin.

Maheshwari, B.L. y H.S. Grewal. 2009. Magnetic treatment of irrigation water: Its effects on vegetable crop yield and water productivity. Agr. Water Manage. 96(8), 1229-1236. Doi:10.1016/j. agwat.2009.03.016

Mahmood, S. y M. Usman. 2014. Consequences of magnetized water application on Maize seed emergence in sand culture. J. Agr. Sci. Tech. 16, 47-55.

Marei, A., D. Rdaydeh, D. Karajeh y N. Abu-Khalaf. 2014. Effect of using magnetic brackish water on irrigated bell pepper crop (Capsicum annuum L.) characteristics in Lower Jordan Valley/West Bank. J. Agr. Sci. Tech. 4, 830-838. Doi: 10.17265/21616256/2014.10.005

Moussa, H.R. 2011. The impact of magnetic water application for improving common bean (Phaseolus vulgaris L.) production. New York Sci. J. 4(6), $15-$ 20.

Pérez, J.P. 2014. Uso de los fertilizantes y su impacto en la producción agrícola. Tesis de maestría. Facultad de Ciencias, Departamento de Biociencias, Universidad Nacional de Colombia, Medellín, Colombia.

Racuciu, M., D. Creanga e I. Horga. 2008. Plant growth under static magnetic field influence. Roman. J. Phys. 53(1-2), 353-359.

Radhakrishnan, R. y B.D.R. Kumari. 2012. Pulsed magnetic field: A contemporary approach offers to enhance plant growth and yield of soybean. Plant Physiol. Bioch. 51, 139-144. Doi: 10.1016/j. plaphy.2011.10.017

Rethinam, P., K. Sivaraman y P.K. Sushama. 1994. Nutrition of turmeric. pp. 477-489. En: Advances in Horticulture. Vol. 9. Plantation and spice crops. Part 1 Malhotra Publishing House, New Delhi, India.

Rochalska, M., K. Grabowska y A. Ziarnik. 2008. Impact of low frequency magnetic fields on yield and quality of sugar beet. Int. Agrophysics 23(2): 163-174 
Rojas A., M. Ramírez, R. Lora, E. Amézquita y L. Sánchez. 1992. Fertilización en diversos cultivos: quinta aproximación. ICA, Ed. Produmedios, Mosquera, Colombia.

Sadanandan, A.K. y S. Hamza. 1996. Response of four turmeric (Curcuma longa L.) varieties to nutrients in an oxisol on yield and curcumin content. J. Plantation Crops 24 (Suppl.) 120-125.
Vashisth, A. y S. Nagarajan. 2010. Effect on germination and early growth characteristics in sunflower (Helianthus annuus) seeds exposed to static magnetic field. J. Plant Physiol. 167(2), 149-156. Doi: 10.1016/j.jplph.2009.08.011

Vasilevski, G. 2003. Perspectives of the application of biophysical methods in sustainable agriculture. Bulg. J. Plant Physiol. 29, 179-186. 